\title{
Reflets
}

Revue ontaroise d'intervention sociale et communautaire

\section{La formation en santé à l'Université Laurentienne : perspectives d'avenir - Entrevue avec Gratien Allaire et Denis Mayer}

\section{Lynn Casimiro}

Volume 8, numéro 1, printemps 2002

La réadaptation : son visage français en Ontario

URI : https://id.erudit.org/iderudit/026370ar

DOI : https://doi.org/10.7202/026370ar

Aller au sommaire du numéro

Éditeur(s)

Reflets : Revue ontaroise d'intervention sociale et communautaire

ISSN

1203-4576 (imprimé)

1712-8498 (numérique)

Découvrir la revue

Citer ce document

Casimiro, L. (2002). La formation en santé à l'Université Laurentienne :

perspectives d'avenir - Entrevue avec Gratien Allaire et Denis Mayer. Reflets, 8(1), 18-25. https://doi.org/10.7202/026370ar

Tous droits réservés (C) Reflets : Revue ontaroise d'intervention sociale et communautaire, 2002
Ce document est protégé par la loi sur le droit d'auteur. L'utilisation des services d'Érudit (y compris la reproduction) est assujettie à sa politique d'utilisation que vous pouvez consulter en ligne.

https://apropos.erudit.org/fr/usagers/politique-dutilisation/ 


\section{La formation en santé à l'Université Laurentienne : perspectives d'avenir Entrevue avec Gratien Allaire et Denis Mayer}

Cette entrevue a été réalisée par Lynn Casimiro. Gratien Allaire est vice-recteur à l'enseigement et à la recherche (affaires francophones) de l'Université Laurentienne et Denis Mayer est directeur général et affaires étudiantes de l'Université Laurentienne. L'entrevue téléphonique s'est déroulée le 22 avril 2002. Nous remercions Messieurs Allaire et Mayer pour leurs propos spontanés et forts intéressants.

Située à Sudbury, l'Université Laurentienne est un établissement d'enseignement bilingue qui dessert de prime abord les communautés du Nord de l'Ontario. En pleine expansion, elle offre déjà des programmes de premier et de deuxième cycle et hébergera bientôt l'école de médecine du Nord, la première à être établie au Canada en 30 ans. Au fils des ans, l'Université Laurentienne a développé une expertise en matière de formation à distance, affichant au-delà de 300 cours utilisant diverses modalités d'enseignement. Par ailleurs, elle compte exploiter les nouvelles technologies pour accroitre le nombre de cours et de programmes offerts à distance.

Nous avons voulu en connaitre davantage sur le désir de l'Université Laurentienne d'augmenter le nombre de programmes relevant des divers domaines de la santé. Aussi, avons-nous discuté avec Gratien Allaire, Vice-recteur à l'enseignement et à la recherche et plus particulièrement des affaires francophones ainsi qu'avec Denis Mayer, Directeur général et affaires étudiantes. Puisque M. Allaire connaît bien sa clientèle et les besoins de sa communauté professionnelle et étudiante, il était le candidat de choix pour nous informer des intentions de l'Université 
Laurentienne en matière de formation en santé. Quant à lui, M. Mayer est un expert en matière de formation à distance. Président de l'Association canadienne d'éducation à distance (ACED), il siège également sur le Groupe de travail sur la médiatisation du Centre national de formation en santé (CNFS). Il possède un bagage important de connaissances au sujet des nouvelles technologies. En effet, on nous dit qu'il est tombé dedans quand il était petit.

Reflets: Quels sont les défis les plus importants auxquels fait face l'Université Laurentienne en matière d'accès à la formation en Sciences de la santé?

G. Allaire: Un des défis dominants en Sciences de la santé est d'élargir la palette de cours et de programmes. Nous offrons déjà un bon choix de cours en Sciences de la santé. Notons particulièrement le baccalauréat en Sciences infirmières, la formation spécialisée des infirmières autorisées et des infirmières praticiennes. L'Université Laurentienne offre également un baccalauréat et une maitrise en Sciences sociales, dont les cours à temps partiel ou à temps complet sont donnés à distance. L'accessibilité est donc très bonne de ce côté. En partenariat avec McMaster et Ryerson, nous avons aussi développé récemment un programme de formation pour les sagesfemmes. L'Université Laurentienne est l'hôtesse du volet français. N'oublions pas le baccalauréat et la maîtrise en activité physique qui comportent des cours en promotion de la santé.

Un deuxième défi se situe sur le plan de la langue. Nous élaborons une nouvelle école de médecine qui a été définie d'abord et avant tout comme une école de langue anglaise. Il s'agit de faire en sorte qu'à l'intérieur de ces initiatives, comme d'ailleurs au niveau de la maîtrise en sciences infirmières qui sont des initiatives en anglais, nous nous assurions que les francophones aient leur place. À des degrés divers, lorsqu'on parle de questions de santé, la dimension linguistique est importante. Ce n'est pas juste un outil de communication; c'est un outil qui permet l'empathie entre le client et le professionnel. Beaucoup plus qu'au niveau des mots, la communication est fondamentale en santé mentale ou en orthophonie, par exemple. Dans de tels cas, la langue est une donnée première. 
Toutefois, le plus important défi est d'assurer que les gens du Nord reçoivent sur place leur formation en santé pour que ces nouveaux professionnels restent ici une fois leurs études complétées. Si ces gens, intéressés par les carrières en santé, sont envoyés vers Ottawa, ou vers le Québec pour obtenir leur formation, il est fort probable qu'ils resteront là où ils ont été formés.

Reflets: Est-ce que l'Université Laurentienne envisage offrir de la formation qui découle du volet de la réadaptation?

G. Allaire et D. Mayer : Oui.

Reflets: Quels sont les défis que vous devez surmonter pour offrir un programme de formation en réadaptation?

G.Allaire: Du coté des professions en santé beaucoup plus spécialisées, physiothérapie, ergothérapie, orthophonie et audiologie, l'un des principaux défis est de recruter des spécialistes prêts à venir enseigner à Sudbury. Il nous faut trouver des incitatifs puissants. C'est une situation plus délicate qu'on aimerait palier à l'aide de modalités d'enseignement à distance en utilisant toutefois des formules pédagogiques à inventer, probablement à partir de celles qu'on connaît déjà, qui nous permettraient un accès à une formation continue et de haute qualité. Les nouvelles technologies figurent à l'avant-plan de cette nouvelle façon de faire.

Au contraire nous ne prévoyons aucune difficulté pour le volet clinique des ces programmes de formation. Nous avons de bons rapports avec les milieux cliniques qui sont très ouverts à ce genre de choses.

Reflets: Alors, si je comprends bien, les défis touchant la formation en réadaptation se résument comme suit:

- attirer des spécialistes pouvant offrir l'enseignement requis

- répondre à la demande des communautés du Nord en matière de professionnels de la santé, en formant des gens chez-vous.

G. Allaire: C'est bien ça! Si on offre des programmes en collaboration, la question se pose de façon un peu différente. Les difficultés de recrutement sont atténuées, bien qu'elles demeurent toujours présentes. 
D. Mayer: Parlons aussi du recrutement des apprenants. Les étudiants potentiels ne pensent pas automatiquement à l'Université Laurentienne lorsqu'il s'agit de formation en santé et plus spécifiquement en réadaptation. Nous devons tailler notre place et nous créer un nom dans ce domaine. C'est donc important que notre école de médecine parte du bon pied parce que toute autre initiative suivra son exemple. Nous voulons que les étudiants du Nord pensent à l'Université Laurentienne pour combler leurs besoins en matière de formation en Sciences de la santé.

Reflets: Hormis la question de recrutement de professionnels, est-ce que c'est possible d'avoir une école de réadaptation à l'Université Laurentienne? Y a-t-il, par exemple, une demande de la part des communautés du Nord de l'Ontario pour des programmes en réadaptation?

G.Allaire: Il y a certains domaines où la communauté en a explicitement fait la demande. C'est le cas de l'orthophonie. En ce qui a trait à la physiothérapie et à l'ergothérapie, je ne suis pas vraiment en mesure de le déterminer. Il est clair que pour assurer la viabilité d'un programme autonome il nous faut une population étudiante assez importante et je ne suis pas certain qu'on l'ait. Par contre si on parle d'un programme qui est offert en collaboration, je pense que nous avons un nombre suffisant d'étudiants.

Dans le cas d'un programme offert en collaboration, il nous faudrait sur place un ou deux spécialistes pour encadrer des étudiants. Évidemment, les cliniciens peuvent jouer un rôle extrêmement important au niveau de la pratique professionnelle et clinique. Toutefois, au niveau académique, c'est important qu'il y ait sur place des professeurs pour répondre aux questions des étudiants.

Reflets: Tantôt, vous avez parlé des technologies pour développer de nouvelles approches pédagogiques et ainsi augmenter l'accès. Pouvez-vous nous donner des détails?

G.Allaire: Le paradigme de développement CNFS auquel nous participons a comme postulat l'utilisation des technologies. Nous travaillons justement avec le consortium du CNFS pour pouvoir offrir en français dans les années à venir de nouveaux programmes en Sciences de la santé. Tantôt, quand nous avons fait allusion aux programmes offerts en collaboration, j'ai renchéri sur le besoin 
de professionnels sur place. Le rôle de la technologie dans ce casci est à mon avis essentiel. L'expérience que nous avons eue avec le programme d'orthophonie nous a donné beaucoup de pistes à poursuivre et d'écueils à éviter.

Reflets: Vous parlez du programme d'orthophonie que vous avez offert en partenariat avec l'Université d'Ottawa.Avez-vous des exemples de pistes ou d'écueils?

G. Allaire: Au niveau des pistes à poursuivre, c'est justement au niveau de l'encadrement. J'insiste sur ce point parce que je considère que c'est la clef du succès lorsqu'on utilise une pédagogie à distance. Au niveau des écueils, je pense qu'il faut une meilleure préparation technique des participants, professeurs et étudiants. Le professeur se retrouve dans des situations d'enseignement différentes. Dans le cas de la vidéoconférence, le professeur doit se préoccuper d'enseigner la matière, il doit se préoccuper des apprenants qui se retrouvent dans une ou plusieurs autres salles de classes et il doit aussi se préoccuper des modalités technologiques. L'étudiant lui, doit pouvoir utiliser la technologie disponible.

Permettez-moi d'offrir cette mise en garde. Bientôt, toutes les disciplines de la réadaptation seront des maîtrises professionnelles. Les étudiants auront développé des habitudes d'apprentissage encore très proches de la salle de classe et le passage à l'utilisation des technologies ne sera pas du tout évident.

Reflets: Très intéressant. Ce que vous avancez, c'est que les technologies vont permettre la création de programmes en partenariat. Mais pour réussir, il sera important de faire évoluer les méthodes d'enseignement et d'apprentissage.

G. Allaire: C'est exactement ça! Présentement, à l'Université Laurentienne, nous développons des standards de développement pour les cours en ligne pour qu'il ne s'agisse pas tout simplement d'une transcription de cours mais bel et bien d'une utilisation maximale des possibilités offertes par les nouvelles technologies. On parle du développement d'une nouvelle pédagogie propre aux technologies utilisées. C'est certain qu'il y a des limites techniques, mais c'est possible de les contourner. Si c'est possible de faire de la consultation médicale sur Internet, je pense que c'est possible 
d'y faire aussi de l'enseignement. Puis, au-delà de l'enseignement, il faut également se préoccuper de l'apprentissage.

D. Mayer: J'aimerais ajouter qu'il faut développer une culture institutionnelle face à l'enseignement via les technologies, parce qu'il y a une grande résistance au changement. Il y a toujours les avant-gardistes qui sont prêts à tout et qui essaient tout. Toutefois, il faut concerter nos efforts. Nous encourageons nos professeurs à utiliser WebCTpour mettre du matériel en ligne. Nous avons également une vingtaine de salles de classe équipées en multimédia, ce qui n'était pas le cas il y a cinq ans. Ces salles de classes permettent une stratégie interactive d'enseignement. Maintenant il faut inciter et former les professeurs à s'en servir. Je me souviens qu'au début on s'en servait pour présenter des vidéos. Ensuite, j'ai noté une utilisation plus fréquente des programmes de présentation tels que Powerpoint. Présentement, plusieurs professeurs se sont créé un site Web et leurs modalités d'enseignement gravitent autour de ce site. On y retrouve des notes de cours et des liens pour guider les étudiants d'un site intéressant à un autre. Je dois admettre qu'à l'Université Laurentienne l'utilisation des technologies a beaucoup changé dans les derniers cinq ans. On a convaincu plusieurs professeurs et on en a apeuré certains. Nous continuons à sensibiliser les gens mais de façon systématique, c'est-à-dire, par département ou par programme. Les Sciences de la santé sont l'un des domaines prioritaires ciblés.

G. Allaire: J'insiste pour dire que l'Université Laurentienne ne veut pas que les technologies ne soient perçues que comme un moyen d'enseignement à distance mais qu'elles soient intégrées en salle de classe. Le but ultime est sans doute que tout professeur puisse communiquer avec des apprenants sur place ou ailleurs sans trop d'efforts supplémentaires. Les modalités et les formules pédagogiques vont sûrement s'établir tranquillement. Du moins c'est ce qu'on espère. L'arrivée des nouvelles technologies fait passer davantage le rôle du professeur vers un facilitateur d'apprentissages, plutôt qu'un enseignant.

Reflets: Revenons un peu à l'expérience de l'Université Laurentienne au niveau du programme conjoint en orthophonie avec l'Université d'Ottawa. 
G.Allaire: C'est intéressant parce que plus on avance, plus on apprend. C'est comme si on avait essayé de reproduire, avec les technologies, ce qui se passait en salle de classe. À mon avis, il aurait fallu développer des stratégies pédagogiques qui collaient mieux aux technologies. Il ne faut pas oublier les difficultés de recrutement et de rétention des professeurs. Nous revenons en fin de compte aux grands défis énumérés au début.

Reflets: Vous participez toutefois avec grand succès, au consortium des institutions offrant le programme des infirmières praticiennes. Les stratégies utilisées sont-elles transférables au domaine de la réadaptation?

D. Mayer: La situation pour le programme d'infirmière praticienne est différente. Chacun des établissements d'enseignement partenaires avait déjà, au départ, une bonne base solide de professionnels en place, lesquels étaient liés à des programmes de formation de base en Sciences infirmières. Il n'y a donc pas eu de problèmes de recrutement et de rétention comme nous l'avons vécu avec le programme d'orthophonie. Le cas a été un peu plus difficile avec le programme des sages-femmes. Nous avons aussi dû faire face à des problèmes de recrutement et de rétention au début de ce programme. La situation s'est heureusement stabilisée depuis. Notons que nos partenaires Ryerson et Mc Master n'ont pas eu à faire face à cette difficulté.

Reflets: Quelles sont vos perspectives d'avenir pour l'Université Laurentienne?

G.Allaire: Notre orientation c'est que les technologies vont nous permettre d'offrir des programmes qu'on ne pourrait offrir autrement. Nous utilisons depuis longtemps les technologies avec généralement des bons résultats et nous avons l'intention de continuer à le faire. La collaboration avec des établissements d'enseignement partenaires sera essentielle dans la présentation de ces nouveaux programmes. J'ajoute que toute offre de nouveaux programmes implique les gouvernements. Il n'y a aucune formation postsecondaire de premier ou deuxième cycle qui peut se donner sans une évaluation et un appui fondamental de leur part.

D. Mayer: Le marché évolue. Peu importe ce que décident les établissements d'enseignement traditionnel, le marché va mettre à la disposition de l'apprenant plusieurs options d'apprentissage d'autant plus 
accessibles. Personne n'aurait pensé avant qu'un apprenant d'Iroquois Falls ou de Kapuskasing pouvait aller étudier à Harvard. Eh bien maintenant c'est possible parce qu'ils ont plusieurs programmes en ligne.Vous pouvez étudier à Deacon en Australie si vous pouvez payer les frais de scolarité. Les universités traditionnelles sont obligées d'examiner les effets de ces nouvelles offres de marché sur leurs effectifs. Elles n'ont pas le choix. Lorsque le Massachusetts Institut of Technology (MIT) a décidé de mettre tous ses cours en ligne, cela a fait un drôle d'effet dans le système d'éducation. Ce ne sont pas tous les apprenants qui souhaitent prendre le virage de la technologie, mais les changements dans le marché indiquent que le consommateur aura davantage de choix. Notre expérience d'enseignement à distance ici à l'Université Laurentienne date de longtemps et elle nous permettra de faire face avec aplomb aux nouvelles tendances.

Reflets: Je vous remercie d'avoir bien voulu partager vos expériences et vos projets avec nous. Je vous félicite pour le travail que vous faites à l'Université Laurentienne et je vous souhaite bon succès. 